\title{
TREES WITH FOUR AND FIVE DISTINCT SIGNLESS LAPLACIAN EIGENVALUES
}

\author{
F. Taghvaee ${ }^{1}$, G.H. Fath-Tabar ${ }^{2}$ \\ ${ }^{1,2}$ Department of Pure Mathematics, Faculty of Mathematical Sciences, \\ University of Kashan, Kashan 87317-53153, I. R. Iran. \\ ${ }^{1}$ taghvaei19@yahoo.com \\ ${ }^{2}$ fathtabar@kashanu.ac.ir
}

\begin{abstract}
Let $G$ be a simple graph with vertex set $V(G)=\left\{v_{1}, v_{2}, \ldots, v_{n}\right\}$ and edge set $E(G)$. The signless Laplacian matrix of $G$ is the matrix $Q=D+A$, such that $D$ is a diagonal matrix and $A$ is the adjacency matrix of $G$. The eigenvalues of $Q$ is called the signless Laplacian eigenvalues of $G$ and denoted by $q_{1}, q_{2}, \ldots, q_{n}$ in a graph with $n$ vertices. In this paper all trees with four and five distinct signless Laplacian eigenvalues are characterized.

Key words and Phrases: Tree, eigenvalue, signless Laplacian matrix, semi-edge walk.
\end{abstract}

\begin{abstract}
Abstrak. Diberikan graf sederhana $G$ dengan himpunan titik $V(G)=$ $\left\{v_{1}, v_{2}, \cdots, v_{n}\right\}$ dan himpunan sisi $E(G)$. Matriks Laplace tak bertanda $G$ adalah matriks $Q=D+A$ dengan $D$ merupakan matriks diagonal yang terindeks himpunan verteks graf $G$ dengan $D_{i i}$ adalah derajat verteks $v_{i}$ dan $A$ adalah matriks ketetanggaan $G$, dengan $A_{i j}=1$ jika ada sisi dari $i$ to $j$ di $G$ dan $A_{i j}=0$ untuk kasus yang lain. Jika $G$ adalah graf dengan $n$ verteks, nilai eigen $Q$ dikatakan Laplace tak bertanda $G$ dan dinotasikan sebagai $q_{1}, q_{2}, \cdots, q_{n}$. Dalam paper ini dibuktikan karakterisasi semua pohon dengan nilai eigen Laplace tak bertanda sebanyak empat dan lima.

Kata kunci: Pohon, matriks Laplace tak bertanda, walk semi-edge.
\end{abstract}

\section{INTRODUCTION}

Let $G$ be a simple graph with vertex set $V(G)=\left\{v_{1}, v_{2}, \ldots, v_{n}\right\}$ and edge set $E(G)$. The adjacency matrix of $G$ is the $\{0,1\}$-matrix $A$ indexed by the vertex set $V(G)$, where $A_{i j}=1$ when there is an edge from $i$ to $j$ in $G$ and $A_{i j}=0$ otherwise. The adjacency spectrum of $G$, denoted by $\operatorname{Spec}(G)$, is the

2010 Mathematics Subject Classification. 05C50, 15 A18.

Received: 18-07-2017, accepted: 17-10-2018. 
multiset of eigenvalues of $A(G)$. The degree matrix of $G$ is defined by $D(G)=$ $\operatorname{diag}\left(d_{G}\left(v_{1}\right), d_{G}\left(v_{2}\right), \ldots, d_{G}\left(v_{n}\right)\right)$, where $d_{G}(v)$ or simply $d(v)$ is the degree of a vertex $v$ in $G$. The signless Laplacian matrix of $G$ is the matrix $Q(G)=D(G)+A(G)$. It is known that $Q(G)$ is nonnegative, symmetric and positive semidefinite, so its eigenvalues are real and can be arranged as $q_{1} \geq q_{2} \geq \ldots \geq q_{n} \geq 0$. We call the eigenvalues of $A(G)$ and $Q(G)$ as the $A$-eigenvalues and $Q$-eigenvalues of $G$, respectively. For additional results on graphs with few distinct $A$-eigenvalues, we refer the reader to $[3,4,6,19,20]$. Recently many studies have been done on the signless Laplacian eigenvalues, the papers $[7,8,9]$ give a survey on this work. Also some bounds for the signless Laplacian eigenvalue can be found in $[10,11]$. The authors $[15,16]$ computed the signless Laplacian spectral moments of some graphs. Let us recall some definitions and notations to be used throughout the paper. The distance between two vertices in a graph is the number of edges in a shortest path connecting them. Denote by $d(u, v)$ the distance between two vertices $u$ and $v$. The diameter $d$ of a graph is the greatest distance between any pair of its vertices.

The line graph of a simple graph $G, L(G)$, is a graph that represents the adjacencies between edges of $G$. In other words the line graph $L(G)$ is a graph such that each vertex of $L(G)$ represents an edge of $G$ and two vertices of $L(G)$ are adjacent if and only if their corresponding edges share a common endpoint ("are incident") in $G$.

Let $G_{1}$ and $G_{2}$ be two graphs. The corona product $G_{1}$ and $G_{2}$, denote by $G_{1} \circ G_{2}$, is obtained by taking one copy of $G_{1}$ and $\left|V\left(G_{1}\right)\right|$ copies of $G_{2}$ by joining any vertex of the $j$-th copy of $G_{2}$ to the $j$-th vertex of $G_{1}$, where $1 \leq j \leq\left|V\left(G_{1}\right)\right|$, see $[2,18]$ for more details.

Throughout this paper we denote a star, a path and a complete graph of order $n$ by $K_{1, n-1}, P_{n}, K_{n}$, respectively. Let $S_{a, b}$ be the double star graph obtained from the stars $K_{1, a}$ and $K_{1, b}$ by joining the vertex of degree $a$ in $K_{1, a}$ and the vertex of degree $b$ in $K_{1, b}$, see Figure 1. In this paper all trees with four and five distinct signless Laplacian eigenvalues are characterized.

\section{PRELIMINARIES}

In this section, we present some useful facts on graphs by given number of distinct $Q$-eigenvalues. The authors, [8] defined a semi-edge walk in a graph as in the following:

A semi-edge walk $W$ of length $k$ in an (undirected) graph $G$ is an alternating sequence $v_{1}, e_{1}, v_{2}, e_{2}, \ldots, v_{k}, e_{k}, v_{k+1}$ of vertices $v_{1}, v_{2}, \ldots, v_{k+1}$ and edges $e_{1}$, $e_{2}, \ldots, e_{k}$ such that for any $i=1,2, \ldots, k$ the vertices $v_{i}$ and $v_{i+1}$ are end-vertices (not necessarily distinct) of the edge $e_{i}$. If $v_{1}=v_{k+1}$, then we say that $W$ is a closed semi-edge walk. If end-vertices of the edge $e_{i}$ are not distinct, then $W$ is called a walk in $G$. Additional results about walk and semi-edge walk can be found in $[13,14,17]$. 
Theorem 2.1. ([5]) Let $Q$ be the signless Laplacian matrix of a graph $G$. Then the $(i, j)$-entry of the matrix $Q^{k}$ is equal to the number of semi-edge walks of length $k$ starting at vertex $v_{i}$ and terminating at vertex $v_{j}$.

Theorem 2.2. ([5]) Let $G$ be a connected graph with diameter $d$. Then $G$ has at least $d+1$ distinct signless Laplacian eigenvalues.

Theorem 2.3. ([5]) The multiplicity of 0 as a signless Laplacian eigenvalue of an undirected graph $G$ equals the number of bipartite connected components of $G$.

Theorem 2.4. ([5] Proposition (1.3.10)) A graph $G$ is bipartite if and only if the Laplacian spectrum and the signless Laplacian spectrum of $G$ are equal.

Theorem 2.5. ([2]) Let $G_{1}$ be any graph, $G_{2}$ be an $r$-regular graph, and $G=$ $G_{1} \circ G_{2}$. Let $\operatorname{Spec}\left(G_{1}\right)=\left(\mu_{1}, \mu_{2}, \ldots, \mu_{n}\right)$ and $\operatorname{Spec}\left(G_{2}\right)=\left(\eta_{1}, \eta_{2}, \ldots, \eta_{m}=r\right)$. Then

(a) $\frac{\left.\mu_{i}+r \pm \sqrt{\left(r-\mu_{i}\right.}\right)^{2}+4 m}{2} \in \operatorname{Spec}(G)$ with multiplicity 1 for $i=1, \ldots, n$.

(b) $\eta_{j} \in \operatorname{Spec}(G)$ with multiplicity $n$ for $j=1, \ldots, m-1$.

In the following we have an explicit formula for the eigenvalues of $L(G)$ in terms of the signless Laplacian eigenvalues of $G$.

Theorem 2.6. ([5]) Suppose $G$ has $m$ edges, and let $q_{1}, q_{2}, \ldots, q_{r}$ be the positive signless Laplacian eigenvalues of $G$. Then the eigenvalues of $L(G)$ are $\mu_{i}=q_{i}-2$ for $i=1, \ldots, r$ and $\mu_{i}=-2$ if $r<i \leq m$.

Corollary 2.7. Let $P_{n}$ be a path with $n$ vertices. Since the path $P_{n}$ has line graph $P_{n-1}$ and is bipartite, the signless Laplacian eigenvalues of $P_{n}$ are $2+2 \cos \frac{\pi i}{n}$, $i=1, \cdots, n$.

Lemma 2.8. ([21] Lemma 4.3.) Let $L\left(S_{a, b}\right)$ be the line graph of double star graph $S_{a, b}$, where the degree of the central vertices is equal to $a$ and $b$. Then for $b \geq a>1$, $L\left(S_{a, b}\right)$ has exactly four distinct eigenvalues.

In [6], the authors discussed on graphs with three distinct $Q$-eigenvalues and they shown that the largest $Q$-eigenvalue of a connected graph $G$ is non integer if and only if $G=K_{n}-e$, for $n \geq 4$. Also the authors in [12], characterized all graphs with four Laplacian eigenvalues and they presented some families of graphs with four distinct Laplacian eigenvalues. 


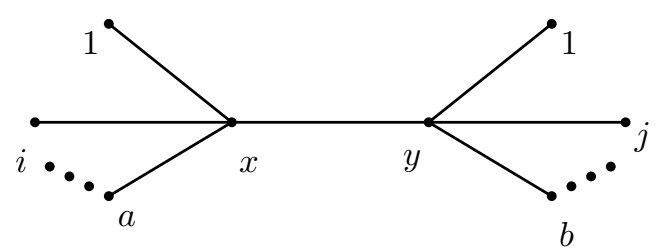

Figure 1. A double star graph.

\section{MAIN RESULTS}

In this section, all trees with four and five distinct signless Laplacian eigenvalues are characterized. To do this we need the following lemma.

Lemma 3.1. The double star graph $S_{a, b}$ with $b \geq a>1$ has exactly five distinct signless Laplacian eigenvalues.

Proof. By Lemma 2.8., the line graph of double star graph $S_{a, b}$ has exactly four distinct eigenvalues. Thus Theorem 2.6. states that the graph $S_{a, b}$ has four positive signless Laplacian eigenvalues. On the other hand by Theorem 2.3, the graph $S_{a, b}$ has 0 as signless Laplacian eigenvalues. So the double star graph $S_{a, b}$ has exactly five signless Laplacian eigenvalues.

In the paper [12], the authors characterized all bipartite graphs with four distinct Laplacian eigenvalues. By Theorem 2.4., all graphs introduced in that paper have four signless Laplacian eigenvalues. In the following we prove that among all trees the path $P_{4}$ is only tree with four signless Laplacian eigenvalues where this graph is the graph $\mathcal{G}(r, s)$ of the paper [12], with $r=s=1$.

Theorem 3.2. Suppose that $G$ is a tree. Then $G$ has exactly four distinct signless Laplacian eigenvalues if and only if $G \cong P_{4}$.

Proof. If $G \cong P_{4}$, then Corollary 2.7 states that $G$ has exactly four distinct signless Laplacian eigenvalues. Conversely let $G$ be a tree with four distinct signless Laplacian eigenvalues. Then by Theorem 2.2., $4 \geq d+1$ and so $d \leq 3$. If $d=2$, then $G \cong S_{n}$. By [1], the star graph $S_{n}$ has three signless Laplacian eigenvalues. Now suppose that $d=3$. Let $i$ and $j$ be the vertices where $d(i, j)=3$ and $i-x-y-j$ be a path between $i$ and $j$. If $G$ just has the same number of vertices, then $G \cong P_{4}$. Now assume that $|V(G)|=n>4$, then the other vertices of $G$ must be connected to $x$ or $y$. Thus the resulting graph is double star graph $S_{a, b}$. By Lemma 3.1. the double star graph $S_{a, b}$ has five distinct signless Laplacian eigenvalues. Therefore only tree with four distinct signless Laplacian eigenvalues is the path $P_{4}$.

We now obtain all trees with exactly five signless Laplacian eigenvalues. For this purpose we consider the following Lemma and then we conclude that only trees with five signless Laplacian eigenvalues are the path $P_{5}$ and the double star graph 


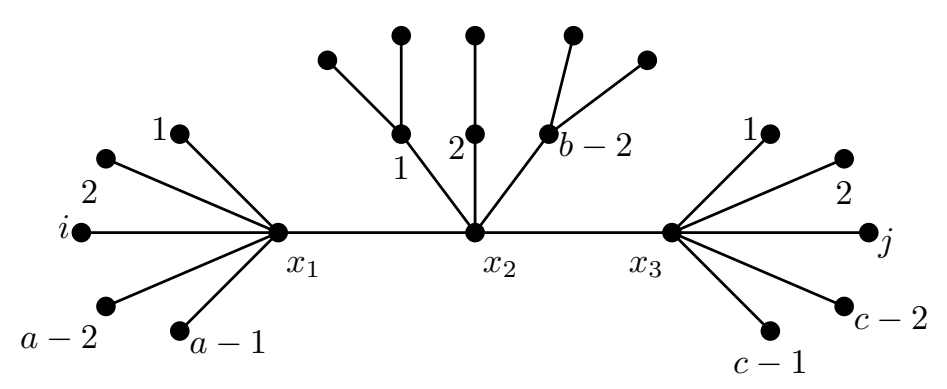

Figure 2. The graph $\mathrm{H}$.

$S_{a, b}$. Let $H$ be the graph where is shown in Figure 2. In following when we say that there is a semi-edge on a vertex, it means that we have a semi-edge walk on the edges connected to that vertex.

Lemma 3.3. For the graph $H$ we have:

- $\left[Q^{5}(H)\right]_{x_{1}, x_{3}}=2 a^{2}-a+2 b^{2}-2 b+2 c^{2}-c+\sum_{i=1}^{b-2} r_{i}+2 a b+2 a c+2 b c+$ $a^{3}+a^{2} b+a^{2} c+b^{2} a+c^{2} a+b^{3}+b^{2} c+c^{2} b+c^{3}+a b c-2$,

- $\left[Q^{4}(H)\right]_{x_{1}, x_{3}}=a^{2}+b^{2}+c^{2}+a+b+c+a b+a c+b c-2$,

- $\left[Q^{3}(H)\right]_{x_{1}, x_{3}}=a+b+c$,

- $\left[Q^{2}(H)\right]_{x_{1}, x_{3}}=1$,

- $[Q(H)]_{x_{1}, x_{3}}=0$.

Proof. Consider the graph $H$ such that $d\left(x_{1}\right)=a, d\left(x_{2}\right)=b$ and $d\left(x_{3}\right)=c$. Denote by $w$ and $s . w$ an edge and semi-edge, respectively. To calculate the $\left(x_{1}, x_{3}\right)$ entry of $Q^{5}(H)$, we should compute the number of semi-edge walks of length five from the vertex $x_{1}$ to the vertex $x_{3}$. To do this, we have two cases: either there is one semi-edge over the some vertices or there are three semi-edge over the some vertices. In the first case, if the semi-edge is on the vertex $x_{1}$, then for any adjacent vertex to $x_{1}$ such as the vertex 1 , we have two types of semi-edges walks as $x_{1}$ s.w $x_{1} \stackrel{w}{-} 1 \stackrel{w}{-} x_{1} \stackrel{w}{-} x_{2} \stackrel{w}{-} x_{3}$ and $x_{1} \stackrel{w}{-} 1 \stackrel{w}{-} x_{1} \stackrel{s \cdot w}{-} x_{1} \stackrel{w}{-} x_{2} \stackrel{w}{-} x_{3}$. The number of each such semi-edge walks is equal to $a^{2}$. Also for any vertex that is adjacent to the vertices $x_{2}$ and $x_{3}$ such as the vertex 1 , we have two types of semi-edges walks as $x_{1} \stackrel{s . w}{-} x_{1} \stackrel{w}{-} x_{2} \stackrel{w}{-} 1 \stackrel{w}{-} x_{2} \stackrel{w}{-} x_{3}$ and $x_{1} \stackrel{s . w}{-} x_{1} \stackrel{w}{-} x_{2} \stackrel{w}{-} x_{3} \stackrel{w}{-} 1 \stackrel{w}{-} x_{3}$. By a simple check one can see that the number of each such semi-edge walks are equal to $a(b-1)$ and $a(c-1)$, respectively. In total if one semi-edge is on the vertex $x_{1}$, then there is $2 a^{2}+a(b-1)+a(c-1)$ semi-edge walk of length five from $x_{1}$ to $x_{3}$. If the semi-edge is over the adjacent vertices to $x_{1}$, then for any adjacent vertex to $x_{1}$ such as the vertex 1 , we have a semi-edge walk of length five as $x_{1} \stackrel{w}{-} 1 \stackrel{s \cdot w}{-} 1 \stackrel{w}{-} x_{1} \stackrel{w}{-} x_{2} \stackrel{w}{-} x_{3}$. The number of such semi-edge walks is equal to $a-1$.

Now assume that the semi-edge is on the vertex $x_{2}$, then for any adjacent vertex to $x_{2}$ such as the vertex 1 , we have two types of semi-edge walks as $x_{1}-$ 
TREES WITH FOUR AND FIVE DISTINCT SIGNLESS LAPLACIAN EIGENVALUES 307 $x_{2} \stackrel{s . w}{-} x_{2} \stackrel{w}{-} 1 \stackrel{w}{-} x_{2} \stackrel{w}{-} x_{3}$ and $x_{1} \stackrel{w}{-} x_{2}-1 \stackrel{w}{-} x_{2} \stackrel{s . w}{-} x_{2}-x_{3}$. It is easy to see that the number of each such semi-edge walks is equal to $b^{2}$. By a simple calculation one can see that for any vertex that is adjacent to the vertices $x_{1}$ and $x_{3}$, except $x_{2}$, there are $b(a-1)$ and $b(c-1)$ semi-edge walks, respectively. Hence the number of semi-edge walks where have one semi-edge on the vertex $x_{2}$ is equal to $2 b^{2}+b(a-1)+b(c-1)$.

Next let the semi-edge be on the adjacent vertices to the vertex $x_{2}$, then for each of $b-2$ vertices that are adjacent to $x_{2}$, such as 1 , there is a semi-edge walk as $x_{1} \stackrel{w}{-} x_{2} \stackrel{w}{-} \stackrel{s . w}{-} 1 \stackrel{w}{-} x_{2}-x_{3}$. Here, the number of such semi-edge walks is equal to $\sum_{i=1}^{b-2} r_{i}$ in which $r_{i}$ is the degree of the $i$-th vertex that is adjacent to $x_{2}$.

Notice that if the semi-edge is on the vertex $x_{3}$, then by a similar calculation there is $2 c^{2}$ semi-edge walk of length five. Also for any vertex that is adjacent to the vertex $x_{1}$ except $x_{2}$ and for any vertex that is adjacent to the vertex $x_{2}$ except $x_{3}$, there are $c(a-1)$ and $c(b-1)$ semi-edge walks, respectively. So in the case that one semi-edge is on the vertex $x_{3}$ there is $2 c^{2}+c(a-1)+c(b-1)$ semi-edge walk of length five. If the semi-edge is on the adjacent vertices to $x_{3}$, then it is easy to see that the number of such semi-edge walks is equal to $c-1$. Therefore in the first case the number of semi-edge walks of length five from $x_{1}$ to $x_{3}$ is equlal to:

$a-1+2 a^{2}+a(b-1)+a(c-1)+\sum_{i=1}^{b-2} r_{i}+2 b^{2}+b(a-1)+b(c-1)+2 c^{2}+c(a-1)+c(b-1)+c-1$.

In the case that there are three semi-edges on some vertices, Table 1 shows the number of semi-edge walks of length five from $x_{1}$ to $x_{3}$.

Finally we have:

$$
\begin{aligned}
{\left[Q^{5}(H)\right]_{x_{1}, x_{3}} } & =2 a^{2}-a+2 b^{2}-2 b+2 c^{2}-c+\sum_{i=1}^{b-2} r_{i}+2 a b+2 a c+2 b c+a^{3} \\
& +a^{2} b+a^{2} c+b^{2} a+c^{2} a+b^{3}+b^{2} c+c^{2} b+c^{3}+a b c-2 .
\end{aligned}
$$

In the following we calculate the $\left[Q^{4}(H)\right]_{x_{1}, x_{3}}$. To do this, we should compute the number of semi-edge walks of length four from the vertex $x_{1}$ to the vertex $x_{3}$. We have two cases: either there is no semi-edge on every vertex or there are two semi-edges on some vertices. Consider the case that there is no semi-edge on the vertices. Then it is easy to check that there are $a, b-1$ and $c-1$ semi-edge walks of length four, for the adjacent vertices to the vertices $x_{1}, x_{2}$ and $x_{3}$, respectively.

In the second case, we have two semi-edges on some vertices. Assume that every two semi-edges are on the vertex $x_{1}$, then a simlpe calculation states that there is $a+a(a-1)$ semi-edge walk of length four from $x_{1}$ to $x_{3}$. While if two semi-edges are on each of the vertices $x_{2}$ and $x_{3}$, then there are $b+b(b-1)$ and $c+c(c-1)$ semi-edge walks of length four, respectively. Now if one semi-edge is on the vertex $x_{1}$ and another is on the vertices $x_{2}$ or $x_{3}$, then by a simple calculation one can see that the number of such semi-edge walks are $a b$ and $a c$, respectively. 
TABLE 1. Some of semi-edge walks of length five and their number.

\begin{tabular}{|c|c|}
\hline Three semi-edges on vertices & The number of semi-edge walks \\
\hline $\begin{array}{c}\text { two semi-edge on } x_{1} \text { and } \\
\text { one semi-edge on } x_{2}\end{array}$ & $a b+a b(a-1)$ \\
\hline $\begin{array}{c}\text { two semi-edge on } x_{1} \text { and } \\
\text { one semi-edge on } x_{3}\end{array}$ & $a c+a c(a-1)$ \\
\hline $\begin{array}{c}\text { one semi-edge on } x_{1} \text { and } \\
\text { two semi-edge on } x_{2}\end{array}$ & $a b+a b(b-1)$ \\
\hline $\begin{array}{c}\text { one semi-edge on } x_{1} \text { and } \\
\text { two semi-edge on } x_{3}\end{array}$ & $a c+a c(c-1)$ \\
\hline $\begin{array}{c}\text { two semi-edge on } x_{2} \text { and } \\
\text { one semi-edge on } x_{3}\end{array}$ & $b c+b c(b-1)$ \\
\hline $\begin{array}{c}\text { one semi-edge on } x_{2} \text { and } \\
\text { two semi-edge on } x_{3}\end{array}$ & $a+3 a(a-1)+a(a-1)(a-2)$ \\
\hline three semi-edge on $x_{1}$ & $b+3 b(b-1)+b(b-1)(b-2)$ \\
\hline three semi-edge on $x_{2}$ & $c+3 c(c-1)+c(c-1)(c-2)$ \\
\hline three semi-edge on $x_{3}$ & $a b c$ \\
\hline $\begin{array}{c}\text { one semi-edge on } x_{1}, \\
\text { one semi-edge on } x_{2} \text { and } \\
\text { one semi-edge on } x_{3}\end{array}$ & \\
\hline
\end{tabular}

Next if one semi-edge is on the vertex $x_{2}$ and another is on the vertex $x_{3}$, then there is $b c$ semi-edge walk of length four. Finally we have:

$$
\left[Q^{4}(H)\right]_{x_{1}, x_{3}}=a^{2}+b^{2}+c^{2}+a+b+c+a b+a c+b c-2 .
$$

To compute the $\left[Q^{3}(H)\right]_{x_{1}, x_{3}}$, we should calculate the number of semi-edge walks of length three from $x_{1}$ to $x_{3}$. Such semi-edge walks occur in the case that we have one semi-edge on some vertices. By a simple check one can see that if the semi-edge is on the vertices $x_{1}, x_{2}$ and $x_{3}$, then there are $a, b$ and $c$ semi-edge walks of length three, respectively. Therefore $\left[Q^{3}(H)\right]_{x_{1}, x_{3}}=a+b+c$. It is easy to see that there is one walk of length two from $x_{1}$ to $x_{3}$ and there is no semi-edge walk of length 1 from $x_{1}$ to $x_{3}$. This implies that $\left[Q^{2}(H)\right]_{x_{1}, x_{3}}=1$ and $[Q(H)]_{x_{1}, x_{3}}=0$. This completes the proof.

Lemma 3.4. The number of closed semi-edge walks of length $k, 1 \leq k \leq 5$, on the vertex $i$ in $H$ are as the following:

- $\left[Q^{5}(H)\right]_{i, i}=a^{3}+4 a^{2}+6 a+b+4$,

- $\left[Q^{4}(H)\right]_{i, i}=a^{2}+3 a+4$,

- $\left[Q^{3}(H)\right]_{i, i}=a+3$,

- $\left[Q^{2}(H)\right]_{i, i}=2$,

- $[Q(H)]_{i, i}=1$.

ProOF. First we compute the number of closed semi-edge walks of length 5 on the vertex $i$. There are three cases: either there is one semi-edge on some vertices, or 

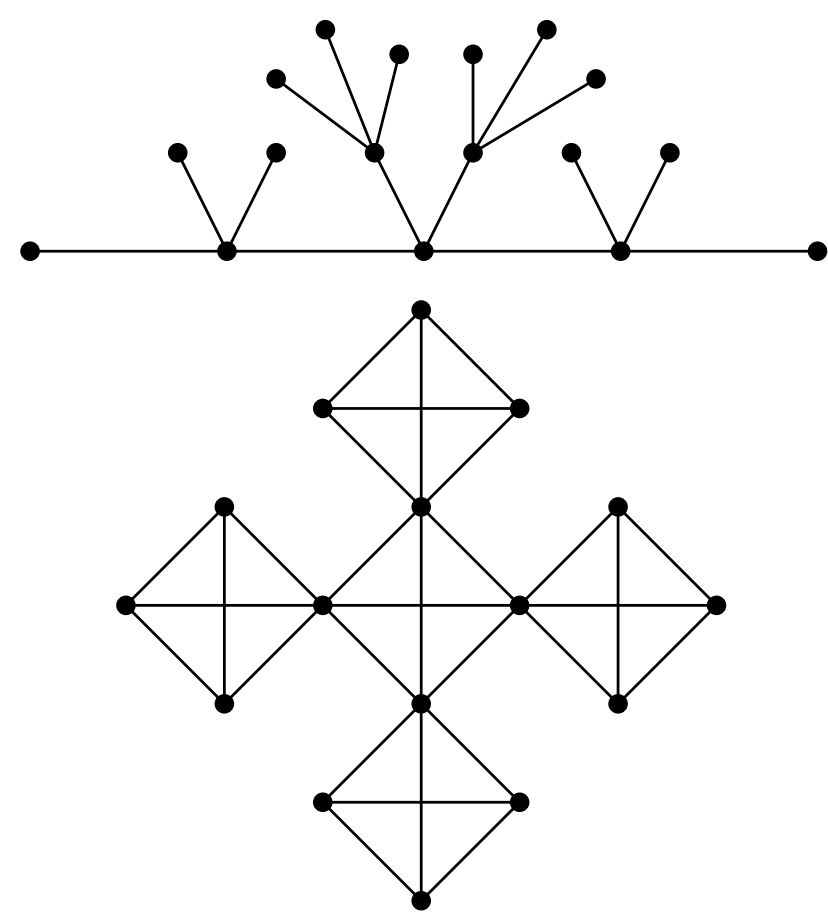

Figure 3. A tree of order 17 and its line graph that is corona product of $K_{4} \circ K_{3}$.

there are three semi-edges on some vertices or there are five semi-edges on vertices. In the first case, if the semi-edge is on the vertex $i$, then a simple calculation states that there is $2 a$ closed semi-edge walk of length five. While if the semi-edge is on the vertex $x_{1}$, there is $2 a^{2}$ closed semi-edge walk of length five. Notice that if the semi-edge is on the adjacent vertices to $x_{1}$, then by a simple check one can see that there is $a+b-1$ closed semi-edge walk.

In the second case, if three semi-edges are on the vertex $i$, then it is easy to see that there are four closed semi-edge walks as following:

$$
\begin{aligned}
& i \stackrel{s . w}{-} i \stackrel{s . w}{-} i \stackrel{s . w}{-} i \frac{w}{-} x_{1}-i, \\
& i \stackrel{s . w}{-} i \frac{s . w}{-} i \frac{w}{-} x_{1}-i \stackrel{s . w}{-} i, \\
& i \stackrel{s . w}{-} i \frac{w}{-} x_{1}-i \stackrel{s . w}{-s . w}-i, \\
& i-x_{1}-i-i \frac{s . w}{-} i \stackrel{s . w}{-} i .
\end{aligned}
$$

Now let two semi-edges be on the vertex $i$ and the third semi-edge be on the vertex $x_{1}$. Then it is easy to see that there is $3 a$ closed semi-edge walk on the vertex $i$. If two semi-edges are on the vertex $x_{1}$ and another is on the vertex $i$, then it is easy 
to check that the number of such closed semi-edge walks is equal to $2 a+2 a(a-1)$. If three semi-edges are on the vertex $x_{1}$, then a simple calculation show that there is $a+3 a(a-1)+a(a-1)(a-2)$ closed semi-edge walk.

In the third case, there is one closed semi-edge walk of length five as $i \stackrel{s . w}{-}$ $i \stackrel{s . w}{-} i \stackrel{s . w}{-} i \stackrel{s . w}{-} i \stackrel{s . w}{-} i$. Therefore the number of closed semi-edge walk of length five on the vertex $i$ is equal to $a^{3}+4 a^{2}+6 a+b+4$.

Now we calculate the number of closed semi-edge walks of length four on the vertex $i$. For any adjacent vertex to $x_{1}$ such as the vertex 1 , there is one semi-edge walk of length four of this form, $i \stackrel{w}{-} x_{1} \stackrel{w}{-} 1 \stackrel{w}{-} x_{1} \stackrel{w}{-} i$. The number of such walk is equal to $a$. In the case that we have two semi-edges on the vertex $x_{1}$, there is $a+a(a-1)$ closed semi-edge walk of length four. Also if two semi-edges are on the vertex $i$, then a simple calculation states that there are three closed semi-edge walks of length four. While if one semi-edge is on the vertex $i$ and another is on the vertex $x_{1}$, then there is $2 a$ closed semi-edge walk of length four. Now assume that there are four semi-edges on the vertex $i$. Then there is one closed semi-edge walk of the form $i \stackrel{s . w}{-} i \stackrel{s . w}{-} i \stackrel{s . w}{-} i \stackrel{s . w}{-} i$. In total the number of closed semi-edge walks of length four is equal to $a^{2}+3 a+4$.

To calculate the $\left[Q^{3}(H)\right]_{i, i}$, it is easy to see that there is one closed semi-edge walk of length three such as $i \stackrel{s . w}{-} i \stackrel{s . w}{-} i \stackrel{s . w}{-} i$. Also if one semi-edge is on the vertex $i$, then there are two types semi-edges of the form $i \stackrel{w}{-} x_{1} \stackrel{w}{-} i \stackrel{s . w}{-} i$ and $i \stackrel{s . w}{-} i \stackrel{w}{-} x_{1} \stackrel{w}{-} i$. Let the semi-edge be on the vertex $x_{1}$. Then by a simple check one can see that there is $a$ closed semi-edge walk of length 3 . Therefore $\left[Q^{3}(H)\right]_{i, i}=a+3$. It is easy to see that there are two closed semi-edge walks of length two and one closed semi-edge walk of length one on the vertex $i$. This completes the proof.

Lemma 3.5. The following relations are satisfied:

- $\left[Q^{5}(H)\right]_{j, j}=c^{3}+4 c^{2}+6 c+b+4,\left[Q^{4}(H)\right]_{j, j}=c^{2}+3 c+4,\left[Q^{3}(H)\right]_{j, j}=c+3$, $\left[Q^{2}(H)\right]_{j, j}=2$ and $[Q(H)]_{j, j}=1$.

- $\left[Q^{5}(H)\right]_{i, j}=a+b+c+2$ and $\left[Q^{4}(H)\right]_{i, j}=\left[Q^{3}(H)\right]_{i, j}=\left[Q^{2}(H)\right]_{i, j}=$ $[Q(H)]_{i, j}=0$.

- $\left[Q^{5}(H)\right]_{i, x_{3}}=a^{2}+b^{2}+c^{2}+2 a+2 b+2 c+a b+a c+b c-1,\left[Q^{4}(H)\right]_{i, x_{3}}=$ $a+b+c+1,\left[Q^{3}(H)\right]_{i, x_{3}}=1$ and $\left[Q^{2}(H)\right]_{i, x_{3}}=[Q(H)]_{i, x_{3}}=0$.

- $\left[Q^{5}(H)\right]_{x_{2}, j}=6 b^{2}+6 c^{2}+3 b c+\sum_{i=1}^{b-2} r_{i}+a-b+b^{3}+b^{2} c+c^{2} b+c^{3}-3 b^{2}+$ $2 b-3 c^{2}+2 c-1,\left[Q^{4}(H)\right]_{x_{2}, j}=b^{2}+c^{2}+b c+2 b+2 c,\left[Q^{3}(H)\right]_{x_{2}, j}=b+c+1$, $\left[Q^{2}(H)\right]_{x_{2}, j}=1$ and $[Q(H)]_{x_{2}, j}=0$.

Proof. The proof of the first part is similar to Lemma 3.4 and the proof of other parts are similar to Lemma 3.3.

What follows, we consider the set all trees and obtain trees with exactly five distinct signless Laplacian eigenvalues. 
Theorem 3.6. A tree $G$ has exactly five distinct signless Laplacian eigenvalues if and only if $G \cong P_{5}$ or $G \cong S_{a, b}$.

Proof. If $G \cong P_{5}$ or $G \cong S_{a, b}$, then Corollary 2.7 and Lemma 3.1 state that $G$ has exactly five distinct signless Laplacian eigenvalues. Conversely suppose that $G$ has $q_{1}, q_{2}, q_{3}, q_{4}$ and $q_{5}$ as the signless Laplacian eigenvalues. Then the minimal polynomial of the matrix $Q$ is $f(x)=\left(x-q_{1}\right)\left(x-q_{2}\right) \ldots\left(x-q_{5}\right)$. So we have the following relation:

$$
\begin{gathered}
Q^{5}-\left(\sum_{i=1}^{5} q_{i}\right) Q^{4}+\left(\sum_{1 \leq i<j \leq 5} q_{i} q_{j}\right) Q^{3}-\left(\sum_{1 \leq i<j<r \leq 5} q_{i} q_{j} q_{r}\right) Q^{2} \\
+\left(\sum_{1 \leq i<j<r<t \leq 5} q_{i} q_{j} q_{r} q_{t}\right) Q-\left(q_{1} q_{2} q_{3} q_{4} q_{5}\right) I=0 .
\end{gathered}
$$

Since $G$ is bipartite, one of these eigenvalues is equal to 0 . Thus the last sentence of the equation (1) is 0 . On the other hand, by Theorem 2.2 we have $d \leq 4$. If $d=2$, then $G \cong S_{n}$ which is a contradiction. If $d=3$, then $G \cong S_{a, b}$ and Lemma 3.1. states that $G$ has five signless Laplacian eigenvalues.

Now assume that $d=4, i$ and $j$ are two vertices such that $d(i, j)=4$ and $i-x_{1}-x_{2}-x_{3}-j$ is a path of length four between $i$ and $j$. If $G$ has only these five vertices, then $G \cong P_{5}$. Otherwise let $|V(G)|=n \geq 6$, then the other vertices must be connected to the vertices $x_{1}, x_{2}$ or $x_{3}$, and so $G=H$. We first claim that in this graph $d\left(x_{1}\right)=d\left(x_{2}\right)=d\left(x_{3}\right)$. By considering equation (1), we have the following system:

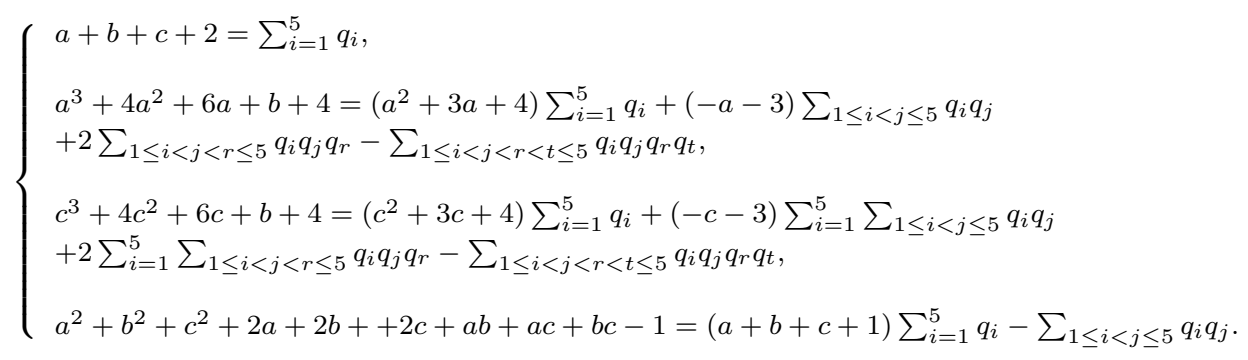

In above system, we subtract the third equation from the second one, next the fourth relation multiplying in $c-a$ and finally we sum both of the resulting relations with above relation. At the rest by substituting the value of $\sum_{i=1}^{5} q_{i}$ of the first equation, we obtain

$$
a=c \text {. }
$$


Now by considerin the $(i, j),\left(x_{1}, x_{3}\right),\left(i, x_{3}\right)$ and $\left(x_{2}, j\right)$-entries of equation $(1)$, we have the following new system:

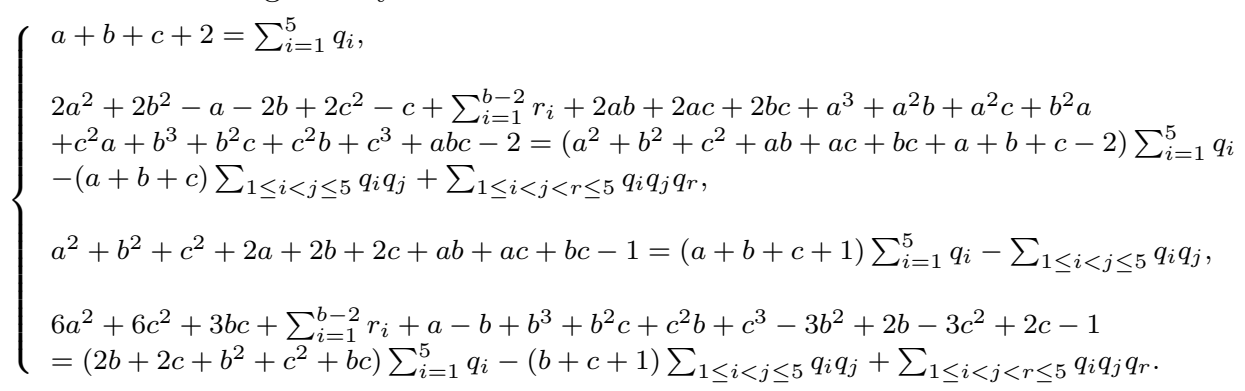

In recent system, first we subtract the fourth equation from the second equation, next the third relation multiplying in $1-a$ and then we sum both of this relation by the above relation. At the rest we replace the value of $\sum_{i=1}^{5} q_{i}$ from the first equation in the resulting equation and so we have

$$
a=b .
$$

By (2) and (3) we have $a=b=c$. By a simple check one can see that the degree of $(b-2)$ vertices attached to $x_{2}$, can not be equal to one. Thus the degree of these vertices is at least two and so the degree oh these vertices is equal to $a$. If $a=b=c=2$, then $G \cong P_{5}$ and so $G$ has five signless Laplacian eigenvalues. Let $a=b=c \geq 3$, then $G=H$ with $d\left(x_{1}\right)=d\left(x_{2}\right)=d\left(x_{3}\right)=a \geq 3$. We claim that this graph has at least six signless Laplacian eigenvalues. To proves, it is sufficient to show that the line graph $L(H)$ has at least five eigenvalues. Put $F=L(H)$. One can see that the graph $F$ is the corona product of $K_{a}$ and $K_{a-1}$, Figure 3. Notice that Theorem 2.5. states that $-1, \frac{a-3 \pm \sqrt{a^{2}+2 a-3}}{2}$ and $\frac{2 a-3 \pm \sqrt{4 a-3}}{2}$ are members of the spectrum of $K_{a} \circ K_{a-1}$. This implies that the graph $L(H)=K_{a} \circ K_{a-1}$ has at least five eigenvalues. On the other hand, Theorem 2.6. states that the graph $H$ has at least six signless Laplacian eigenvalues. Overall for any $a, b$ and $c$, the graph $H$ has at least six signless Laplacian eigenvalues. Finally we conclude that the trees with exactly five signless Laplacian eigenvalues are the graphs $P_{5}$ and $S_{a, b}$. This completes the proof.

Acknowledgement. The authors are very thankfull from professor Ali Reza Ashrafi for some discussion on this problem. The research of this paper is partially supported by the University of Kashan under grant no 504631/12.

\section{REFERENCES}

[1] Ayoobi, F., Omidi, G.R. and Tayfeh-Rezaie B., A note on graphs whose signless Laplacian has three distinct eigenvalues, Linear Multilinear Algebra, 59 (2011), 701-706.

[2] Barik, S., Pati, S. and Sarma, B.K., The spectrum of the corona of two graphs, SIAM J. Discrete Math., 21 (2007), 47-56. 
[3] Biggs, N., Algebraic Graph Theory, Cambridge Univ, Press, Cambridge 1974.

[4] Bridges, W.G. and Mena, R.A., Multiplicative cones - a family of three eigenvalue graphs, Aequationes Math., 22 (1981), 208-214.

[5] Brouwer, A.E. and Haemers, W.H., spectra of graph, Springer, New York, etc. 2012.

[6] Chuang, H. and Omidi, G.R., Graphs with three distinct eigenvalues and largest eigenvalue less than 8, Linear Algebra Appl., 430 (2009), 2053-2062.

[7] Cvetković, D., New theorems for signless Laplacian eigenvalues, Bull. Cl. Sci. Math. Nat. Sci. Math., 137 (2008), 131-146.

[8] Cvetković, D., Rowlinson, P. and Simić, S.K., Signless Laplacians of finite graphs, Linear Algebra Appl., 423 (2007), 155-171.

[9] Cvetković, D. and Simić, S.K., Towards a spectral theory of graphs based on the signless Laplacian, II, Linear Algebra Appl., 432 (2010), 2257-2272.

[10] Ellahi, H.R., Fath-Tabar, G.H., Gholami, A. and Nasiri, R., On maximum signless Laplacian Estrada index of graphs with given parameters, Ars Math. Contemp., 11 (2016), 381-389.

[11] Fan, Y.Z., Tam, B.S. and Zhou, J., Maximizing spectral radius of unoriented Laplacian matrix over bicyclic graphs of a given order, Linear Multilinear Algebra, 56 (2008), 381-397.

[12] Mohammadian, A. and Tayfeh-Rezaie, B., Graphs with four distinct Laplacian eigenvalues, J. Algebraic Combin., 34 (2011), 671-682.

[13] Taghvaee, F. and Ashrafi, A.R., Comparing fullerenes by spectral moments, J. Nanosci. Nanotechnol., 16 (2016), 1-4.

[14] Taghvaee, F. and Ashrafi, A.R., On spectrum of $I$-graphs and its ordering with respect to spectral moments, Le Matematiche, 16 (2017), 61-75.

[15] Taghvaee, F. and Fath-Tabar, G.H., Signless Laplacian spectral moments of graphs and ordering some graphs with respect to them, Alg. Struc. Appl., 1 (2014), 133-141.

[16] Taghvaee, F. and Fath-Tabar, G.H., On the skew spectral moments of graphs, Trans. Comb., 6 (2017), 47-54.

[17] Taghvaee, F. and Fath-Tabar, G.H., Relationship between coefficients of characteristic polynomial and matching polynomial of regular graphs and its applications, Iranian J. Math. Chem., 8 (2017), 7-23.

[18] Tavakoli, M., Rahbarnia, F. and Ashrafi, A.R., Studying the corona product of graphs under some graph invariants, Trans. Comb., 3 (2014), 43-49.

[19] van Dam, E.R., Regular graphs with four eigenvalues, Linear Algebra Appl., 226/228 (1995), 139-162.

[20] van Dam, E.R., Graphs with few eigenvalues, an interplay between combinatorics and algebra, Center Dissertation Series 20, Thesis, Tilburg University 1996.

[21] Wang, J., Shen, Y. and Huang, Q., Notes on graphs with least eigenvalue at least -2 , Electron. J. Linear Algebra, 23 (2012), 387-396. 